SUNY-NTG-99/8

\title{
The Spectral Density of the QCD Dirac Operator and Patterns of Chiral Symmetry Breaking
}

\author{
D. Toublan and J.J.M. Verbaarschot \\ Department of Physics and Astronomy, SUNY, Stony Brook, New York 11794
}

\begin{abstract}
We study the spectrum of the QCD Dirac operator for two colors with fermions in the fundamental representation and for two or more colors with adjoint fermions. For $N_{f}$ flavors, the chiral flavor symmetry of these theories is spontaneously broken according to $S U\left(2 N_{f}\right) \rightarrow S p\left(2 N_{f}\right)$ and $S U\left(N_{f}\right) \rightarrow O\left(N_{f}\right)$, respectively, rather than the symmetry breaking pattern $S U\left(N_{f}\right) \times S U\left(N_{f}\right) \rightarrow S U\left(N_{f}\right)$ for QCD with three or more colors and fundamental fermions. In this paper we study the Dirac spectrum for the first two symmetry breaking patterns. Following previous work for the third case we find the Dirac spectrum in the domain $\lambda \ll \Lambda_{\mathrm{QCD}}$ by means of partially quenched chiral perturbation theory. In particular, this result allows us to calculate the slope of the Dirac spectrum at $\lambda=0$. We also show that for $\lambda \ll 1 / L^{2} \Lambda_{Q C D}$ (with $L$ the linear size of the system) the Dirac spectrum is given by a chiral Random Matrix Theory with the symmetries of the Dirac operator.
\end{abstract}

PACS: 11.30.Rd, 12.39.Fe, 12.38.Lg, 71.30.+h

Keywords: QCD Dirac operator; Chiral random matrix theory; Partially quenched chiral perturbation theory; Microscopic spectral density; Valence quark mass dependence 


\section{Introduction}

Perhaps the most important reason for the current interest in the infrared sector of the Euclidean Dirac spectrum is its relation with the chiral condensate by means of the Banks-Casher formula [1]. In this way the properties of the smallest eigenvalues of the Dirac operator may be related to the mechanisms of chiral symmetry breaking and to the nature of the chiral phase transition as observed in lattice QCD simulations [2, 3]. However, this is not the only reason for our interest in this part of the Dirac spectrum. What may be more important ultimately is that it is one of the few examples where exact nonperturbative analytical results can be derived from QCD.

One of the main objectives of this paper is to study the QCD Dirac spectrum in the domain $\lambda \ll \Lambda_{\mathrm{QCD}}$. In particular we calculate the slope of the Dirac spectrum near $\lambda=0$. This problem was first addressed in a paper by Smilga and Stern [4. They studied the slope of the Dirac spectrum for the case of three or more colors with fermions in the fundamental representation. In this article we extend previous results [4, 5, 6] to the case of QCD with two colors and fundamental fermions and QCD with adjoint fermions. Before discussing our strategy let us first summarize the approach followed by Smilga and Stern. They studied the scalar susceptibility which, in terms of the spectral density averaged over the Euclidean QCD action,

$$
\rho(\lambda)=\sum_{k}\left\langle\delta\left(\lambda-\lambda_{k}\right)\right\rangle
$$

is given by

$$
K^{a b}=-\delta^{a b} \int_{0}^{\infty} \frac{\rho(\lambda)\left(m^{2}-\lambda^{2}\right)}{\left(m^{2}+\lambda^{2}\right)^{2}} d \lambda .
$$

Their idea was to derive $K^{a b}$ from chiral perturbation theory and invert this relation to obtain information on the Dirac spectrum. Because of the implicit mass dependence of $\rho(\lambda)$ via the fermion determinant in the statistical average over gauge field configurations such inversion is not possible in general. They found that

$$
K^{a b} \sim \delta^{a b} \frac{N_{f}^{2}-4}{N_{f}} \log (m / \Lambda),
$$

where $\Lambda$ is the momentum cutoff of the one-loop integral. Their crucial observation was that the contribution to the integral (2) from the chiral logarithms in $\rho(0)$ cancels because

$$
\int_{0}^{\infty} \frac{\rho(0)\left(m^{2}-\lambda^{2}\right)}{\left(m^{2}+\lambda^{2}\right)^{2}} d \lambda=0 .
$$

Barring any more exotic $\lambda$ dependence of $\rho(\lambda)$ such as for example $\sqrt{m \lambda} \log m$, the logarithmic mass dependence of the scalar susceptibility results entirely from the linear term in $\rho(\lambda)$. 
However, there is another approach to extract the Dirac spectrum directly from the low energy effective partition function which does not suffer from the above mentioned ambiguities in the mass dependence [7, 5, 6]. The starting point of this approach is the so called partially quenched partition function defined as [8, 9, 10]

$$
Z^{\mathrm{pq}}\left(m_{v}, J\right)=\int[d A] \frac{\operatorname{det}\left(\not D+m_{v}+J\right)}{\operatorname{det}\left(\not D+m_{v}\right)} \prod_{f=1}^{N_{f}} \operatorname{det}\left(\not D+m_{s}\right) e^{-S_{Y M}[A]}
$$

where $\not D$ is the Euclidean Dirac operator and $S_{Y M}[A]$ is the Euclidean Yang-Mills action. The valence quark mass dependence of the chiral condensate is then given by [11, 07, 12]

$$
\Sigma\left(m_{v}\right)=\frac{1}{V}\left\langle\sum_{k} \frac{1}{m_{v}+i \lambda_{k}}\right\rangle=\left.\frac{1}{V} \partial_{J}\right|_{J=0} \log Z^{\mathrm{pq}}\left(m_{v}, J\right) .
$$

The spectral density then follows from the discontinuity across the imaginary axis

$$
\rho(\lambda) / V=\frac{1}{2 \pi}(\Sigma(i \lambda+\epsilon)-\Sigma(i \lambda-\epsilon)) .
$$

The valence quark mass dependence of the partition function (5) can be obtained from its corresponding low-energy effective partition function [13, 9, 10, 14] which is based on the specific pattern of chiral symmetry breaking. In this way it is possible to extract exact analytical information on the QCD Dirac spectrum in the domain near zero virtuality. This approach was first followed in [5, 6] for three or more colors with fundamental fermions where in addition to extending the result of Smilga and Stern we were able to calculate the spectral density of the Dirac operator on the scale of the smallest eigenvalues. In this paper we apply this approach to QCD with two colors with fermions in the fundamental representation and QCD with adjoint fermions.

A much simpler theory with the global symmetries of the QCD partition function is chiral Random Matrix Theory (chRMT) [15, 16]. In this theory the matrix elements of the Dirac operator are replaced by Gaussian distributed random variables. It turns out that this does not affect the pattern of chiral symmetry breaking. The separation into three different symmetry classes can be related naturally to the Dyson index of the Dirac operator denoted by the symbol $\beta$. Its value is $\beta=1$ for QCD with two colors and fermions in the fundamental representation, it is $\beta=2$ for QCD with three or more colors and fermions in the fundamental representation, and it is $\beta=4$ for QCD with adjoint fermions. The matrix elements of the Dirac matrix of the corresponding chRMT are real, complex and quaternion real, respectively [16, 56]. Within chRMT it is possible to obtain exact analytical expressions for the spectral density and all spectral correlation functions. As we will see in the next paragraph it is possible to establish a domain where the spectral correlations of the QCD Dirac operator are given by chRMT. Alternatively, the existence of such domain can be understood by means of universality arguments 17, 18, 19, 20, 21, 22, 23, 24. 
We can distinguish three important scales in the QCD Dirac spectrum. They are most conveniently discussed in terms of the the valence quark mass. The first scale is the smallest nonzero eigenvalue of the Dirac operator which is of the order of the average level spacing and is given by

$$
\lambda_{\min } \sim \frac{\pi}{\Sigma_{0} V},
$$

where $\Sigma_{0}$ is the chiral condensate in the chiral limit and $V=L^{4}$ is the volume of spacetime. The second scale corresponds to the valence quark mass for which the Compton wave length of the associated Goldstone boson is equal to the size of the box [7, 25, 5],

$$
\frac{m_{v} \Sigma_{0}}{F^{2}} \sim \frac{1}{L^{2}}
$$

As was argued in [7, 25, 5, 6] the valence quark mass dependence of the chiral condensate is described by chRMT for

$$
m_{v} \ll m_{c}=\frac{F^{2}}{\Sigma_{0} L^{2}} .
$$

The spectral density of the Dirac operator, given by the discontinuity of $\Sigma\left(m_{v}\right)$, is therefore described by chRMT as well in this domain. These results have been confirmed by numerous lattice QCD simulations [26, 0, 27, 28, 29, 30, 31, 32] [33, 34, 35, 36, 37, 38, 39, 40, instanton liquid simulations [41, 25, 42] and other calculations [43, 44]. For valence quark masses below this scale the kinetic term in the effective Lagrangian can be ignored. In [5, 6] it was shown that in this regime the valence quark mass dependence that follows from partially quenched chiral perturbation theory coincides with the result from chRMT. The dimensionless ratio of the scales (8) and (10)

$$
\frac{m_{c}}{\lambda_{\min }} \sim \frac{F^{2} L^{2}}{\pi}
$$

represents the approximate number of eigenvalues that are described by chRMT. A scale analogous to $m_{c}$ occurs in mesoscopic physics where it is known as the Thouless energy 445, 46].

Based on the scales

$$
\lambda_{\min } \ll m_{c} \ll \Lambda_{Q C D}
$$

we thus can distinguish four different domains. With names borrowed from mesoscopic physics, these scales separate the quantum domain, the ergodic domain, the diffusive domain and the ballistic domain. The valence quark masses can be interpreted in terms of a conjugate time. Then $\hbar / m_{c}$ is the time scale for which a particle diffuses over the length of the system with diffusion constant given by $F^{2} / \Sigma_{0}$ [47, 25, 48]. For more 
discussion and additional references on impurity scattering in disordered systems and the Thouless energy we refer to 49, 50, 51.

In the domain of validity for chRMT it is natural to study the spectral density in units of the average level spacing. The corresponding microscopic spectral density is defined by 15

$$
\rho_{s}(u)=\lim _{V \rightarrow \infty} \frac{1}{\Sigma V} \rho\left(\frac{u}{\Sigma V}\right) .
$$

In this paper, we will show by means of a perturbative calculation that in the ergodic domain the microscopic spectral density that follows from partially quenched chiral perturbation theory coincides with the result obtained from the chiral Random Matrix Theory in the same universality class. For smaller values of $m_{v}$ the super-integrations have to be performed exactly. This has been achieved [5, 6] for the case $\beta=2$ which is mathematically much simpler than the cases $\beta=1$ and $\beta=4$ [49, 52, 53] and complete agreement with chRMT was found. It would be interesting to perform a similar calculation for the other two cases as well.

As second important result of this paper is the derivation of the slope of the Dirac spectrum for QCD with two colors and fundamental fermions and for QCD with adjoint fermions. We will derive this result in two different ways. First, according to the method of Smilga and Stern, and second by means of the partially quenched QCD partition function. We find that both methods agree thereby excluding a more exotic mass dependence of the spectral density which would be allowed by the Smilga-Stern approach. Our result for the slope of the Dirac spectrum for $N_{f}$ massless fermions can be summarized as

$$
\rho^{\prime}(\lambda=0)=\frac{\Sigma_{0}^{2}}{16 \pi^{2} F^{4}} \frac{\left(N_{f}-2\right)\left(N_{f}+\beta\right)}{\beta N_{f}},
$$

where $\Sigma_{0}$ is the chiral condensate, $F$ is the pion decay constant and $\beta$ is the Dyson index of the Dirac operator.

In the next two sections we study the symmetry breaking patterns for $\beta=1$ and $\beta=4$. The slope of the Dirac spectrum for these two cases is calculated from standard ChPT in section 4. Partially quenched chiral perturbation theory based on supersymmetry is constructed in section 5. Within these effective theories we calculate the valence quark mass dependence of the chiral condensate. The computations for two colors with fundamental fermions and adjoint fermions are presented separately in sections 6 and 7 , respectively. Some interesting limiting cases and compact expressions for all $\beta$ are given in section 8. In section 9 we show that our results agree with chRMT in the ergodic domain. We also find the Dirac spectral density for larger eigenvalues, in the diffusive domain, and obtain an expression for its slope. Concluding remarks are made in section 10 . 


\section{Symmetries of the QCD Partition Function with Two Colors and Funda- mental Fermions}

The QCD partition function for two colors and fundamental fermions is special in the sense that it is invariant under a larger symmetry group than is the case for more colors. This well-known fact for QCD with fundamental fermions relies on the pseudo-real nature of $S U_{\mathrm{C}}(2)$ [54, 55, 56], and can be easily generalized to the supersymmetric case [57] relevant to the partially quenched QCD partition function. The discussion in this section is mainly included for pedagogical reasons and part of it was already given in reference [58].

The fermionic action is obtained by writing the determinant in the partition function as a Gaussian integral over the Grassmann fields $\phi$ and $\bar{\phi}$. Using the chiral representation of the $\gamma$ matrices with

$$
\gamma_{\mu}=\left(\begin{array}{cc}
0 & \hat{\sigma}_{\mu} \\
\hat{\sigma}_{\mu}^{+} & 0
\end{array}\right)
$$

and $\hat{\sigma}_{\mu}=\left(1, i \sigma_{k}\right)$ with $\sigma_{k}$ the Pauli $\sigma$-matrices, the fermionic action can be written as

$$
S_{F}=\int d^{4} x \sum_{f=1}^{N_{f}}\left(\begin{array}{c}
\bar{\phi}_{R} \\
\bar{\phi}_{L}
\end{array}\right)\left(\begin{array}{cc}
m_{f} & \hat{\sigma}_{\mu}\left(\partial_{\mu}+i A_{\mu}\right) \\
\hat{\sigma}_{\mu}^{+}\left(\partial_{\mu}+i A_{\mu}\right) & m_{f}
\end{array}\right)\left(\begin{array}{c}
\phi_{R} \\
\phi_{L}
\end{array}\right) \text {. }
$$

For $N_{c}=2$, we have that $A_{\mu}^{T}=-\tau_{2} A_{\mu} \tau_{2}$ (with $\tau_{2}=\sigma_{2}$ in color space). Combining this with the relation $\hat{\sigma}_{\mu}^{*}=\sigma_{2} \hat{\sigma}_{\mu} \sigma_{2}$ we find

$$
\bar{\phi}_{L}^{f} \hat{\sigma}_{\mu}^{+}\left(\partial_{\mu}+i A_{\mu}\right) \phi_{R}^{f}=\phi_{R}^{f} \sigma_{2} \tau_{2} \hat{\sigma}_{\mu}\left(\partial_{\mu}+i A_{\mu}\right) \sigma_{2} \tau_{2} \bar{\phi}_{L}^{f},
$$

where we have used that $\partial_{\mu}$ is anti-Hermitian and that the fermion fields are anticommuting Grassmann variables. The fermionic action can thus be rewritten as

$$
S_{F}\left(m_{f}=0\right)=\int d^{4} x \sum_{f=1}^{N_{f}}\left(\begin{array}{c}
\bar{\phi}_{R}^{f} \\
\sigma_{2} \tau_{2} \phi_{R}^{f}
\end{array}\right)\left(\begin{array}{cc}
\hat{\sigma}_{\mu}\left(\partial_{\mu}+i A_{\mu}\right) & 0 \\
0 & \hat{\sigma}_{\mu}\left(\partial_{\mu}+i A_{\mu}\right)
\end{array}\right)\left(\begin{array}{c}
\phi_{L}^{f} \\
\sigma_{2} \tau_{2} \bar{\phi}_{L}^{f}
\end{array}\right) .
$$

Obviously the symmetry group is $G l\left(2 N_{f}\right)$. If the number of left-handed modes is not equal to the number of right-handed modes because of the anomaly, an axial $U(1)$ is broken explicitly as for three or more colors.

The mass term is given by

$$
\begin{aligned}
S_{m} & =\int d^{4} x \sum_{f=1}^{N_{f}}\left[\left(\begin{array}{c}
\phi_{L}^{f} \\
\sigma_{2} \tau_{2} \bar{\phi}_{L}^{f}
\end{array}\right)\left(\begin{array}{cc}
0 & -\frac{1}{2} m_{f} \sigma_{2} \tau_{2} \\
\frac{1}{2} m_{f} \sigma_{2} \tau_{2} & 0
\end{array}\right)\left(\begin{array}{c}
\phi_{L}^{f} \\
\sigma_{2} \tau_{2} \bar{\phi}_{L}^{f}
\end{array}\right)\right. \\
& \left.+\left(\begin{array}{c}
\bar{\phi}_{R}^{f} \\
\sigma_{2} \tau_{2} \phi_{R}^{f}
\end{array}\right)\left(\begin{array}{cc}
0 & \frac{1}{2} m_{f} \sigma_{2} \tau_{2} \\
-\frac{1}{2} m_{f} \sigma_{2} \tau_{2} & 0
\end{array}\right)\left(\begin{array}{c}
\bar{\phi}_{R}^{f} \\
\sigma_{2} \tau_{2} \phi_{R}^{f}
\end{array}\right)\right] .
\end{aligned}
$$


Also in this case we expect a maximum spontaneous breaking of chiral symmetry consistent with the Vafa-Witten theorem [59]. This means that only the subgroup of $U\left(2 N_{f}\right)$ that leaves both $\bar{\phi}_{R} \phi_{R}$ and $\bar{\phi}_{L} \phi_{L}$ invariant remains unbroken. Therefore, only the subgroup that leaves

$$
\left(\begin{array}{c}
\phi_{L}^{f} \\
\bar{\sigma}_{2} \tau_{2} \bar{\phi}_{L}^{f}
\end{array}\right)\left(\begin{array}{cc}
0 & -\sigma_{2} \tau_{2} \\
\sigma_{2} \tau_{2} & 0
\end{array}\right)\left(\begin{array}{c}
\phi_{L}^{f} \\
\sigma_{2} \tau_{2} \bar{\phi}_{L}^{f}
\end{array}\right)
$$

invariant remains unbroken $\left(\bar{\phi}_{R} \phi_{R}\right.$ is invariant under the same transformations). This is the symplectic group $S p\left(2 N_{f}\right)$. The Goldstone manifold is thus given by the coset $S U\left(2 N_{f}\right) / S p\left(2 N_{f}\right)$.

An analogous argument can be made for bosonic quarks. In that case it is essential to allow only symmetry transformations that leave the integrals in the partition function (5) convergent. In that case one finds [57] that the chiral symmetry is broken according to $G l(2, R) \rightarrow O(2)$.

The symmetries of the partially quenched partition function for two colors with fundamental matter fields can be obtained along similar lines. One easily convinces oneself that the symmetry of the Lagrangian is enhanced to $G l\left(2 N_{f}+2 \mid 2\right)$ from the usual group $G l\left(N_{f}+1 \mid 1\right) \times G l\left(N_{f}+1 \mid 1\right)$ for more than two colors. For convergence reasons the symmetry group has to be restricted to a non-compact group $G l(2, R)$ for the bosonic quarks. This symmetry is broken spontaneously by the formation of a chiral condensate in the boson-boson sector and the fermion-fermion sector exactly as discussed above. The remaining symmetry group combines into the ortho-symplectic graded Lie group $\operatorname{OSp}\left(2 N_{f}+2 \mid 2\right)$. The Goldstone manifold is therefore given by [57] the maximum Riemannian submanifold of the coset space $G l\left(2 N_{f}+2 \mid 2\right) / O S p\left(2 N_{f}+2 \mid 2\right)$. This results in a integration domain for the the fermion-fermion block given by the symmetric space $U\left(2 N_{f}\right) / S p\left(2 N_{f}\right)$ and an integration domain for the boson-boson block given by the symmetric space $G l(2, R) / O(2)$.

An explicit parameterization of this Goldstone manifold is given by

$$
V^{T} G V
$$

where

$$
G=\left(\begin{array}{ccc}
0 & 1_{N_{f}+1} & 0 \\
-1_{N_{f}+1} & 0 & 0 \\
0 & 0 & 1_{2}
\end{array}\right)
$$

and $V$ is parameterized as

$$
V=\left(\begin{array}{ccc}
U_{F F} & \alpha_{1} & \alpha_{2} \\
\beta_{1} & a & b \\
\beta_{2} & c & d
\end{array}\right)
$$


Here, $\alpha_{i}$ and $\beta_{i}$ are Grassmann variables, $U_{F F}$ is an $\left(2 N_{f}+2\right) \times\left(2 N_{f}+2\right)$ unitary matrix and $a, b, c$ and $d$ are real numbers.

\section{Symmetries of the QCD Partition Function for Adjoint Fermions}

For QCD with fermions in the adjoint representation the Dirac operator is given by

$$
\not D=\gamma_{\mu} \partial_{\mu}+f^{a b c} \gamma_{\mu} A_{a \mu}
$$

where the $f^{a b c}$ denote the structure constants of the gauge group. In this case, the operator $\gamma_{2} \gamma_{4}(\not D+m)$ is antisymmetric and therefore the square root of the fermion determinant can be represented as a Grassmann integral [60] in the following way

$$
\begin{aligned}
& \operatorname{det}^{1 / 2}\left(\gamma_{2} \gamma_{4}(D+m)\right)= \\
& \int d \psi_{R} d \psi_{L} \exp \left[\left(\begin{array}{c}
\psi_{R}^{f} \\
\psi_{L}^{f}
\end{array}\right) \gamma_{2} \gamma_{4}\left(\begin{array}{cc}
m & \sigma_{\mu}\left(\partial_{\mu}+f^{a b c} \gamma A_{a \mu}\right) \\
\sigma_{\mu}^{+}\left(\partial_{\mu}+f^{a b c} \gamma A_{a \mu}\right) & m
\end{array}\right)\left(\begin{array}{c}
\psi_{R}^{f} \\
\psi_{L}^{f}
\end{array}\right)\right]
\end{aligned}
$$

For simplicity we will consider only even $N_{f}$. The flavor symmetry group is $S U\left(N_{f}\right)$ which acts on the Majorana fields as

$$
\left(\begin{array}{c}
\psi_{R} \\
\psi_{L}
\end{array}\right) \rightarrow\left(\begin{array}{cc}
U & 0 \\
0 & U^{-1}
\end{array}\right)\left(\begin{array}{l}
\psi_{R} \\
\psi_{L}
\end{array}\right)
$$

This symmetry is broken by the chiral condensate which, with maximum breaking of chiral symmetry consistent with the Vafa-Witten theorem, is given by (the color index is denoted by $i$ and the spinor index by $\alpha$ )

$$
\Sigma_{0}=\frac{2}{N_{f}} \sum_{f=1}^{N_{f}}\left\langle\epsilon_{\alpha \beta}\left(\psi_{R \alpha i}^{f} \psi_{R \beta i}^{f}+\psi_{L \alpha i}^{f} \psi_{L \beta i}^{f}\right)\right\rangle,
$$

and is invariant under an $O\left(N_{f}\right)$ subgroup of $S U\left(N_{f}\right)$. The Goldstone manifold in this case is thus given by $S U\left(N_{f}\right) / O\left(N_{f}\right)$.

The square root of an anti-symmetric matrix cannot be represented as an integral over real bosonic variables. Therefore, the above construction fails for bosonic quarks. However, it is possible to represent the inverse determinant as an integral over complex variables. If we use that

$$
\bar{\phi}_{L} \hat{\sigma}_{\mu}^{+}\left(\partial_{\mu}+f^{a b c} \gamma_{\mu} A_{a \mu}\right) \phi_{R}=\left(\sigma_{2} \phi_{R}\right) \hat{\sigma}_{\mu}\left(\partial_{\mu}+f^{a b c} \gamma_{\mu} A_{a \mu}\right) \sigma_{2} \bar{\phi}_{L},
$$

we observe that the flavor symmetry group of the bosonic quarks is doubled. In this case it turns out [57 that the chiral symmetry is broken according to $U^{*}(2, R) \rightarrow S p(2)$. 
The symmetry group of the partially quenched Lagrangian for matter fields in the adjoint representation is $G l\left(N_{f}+2 \mid 2\right)$. Because of convergence reasons only a $U^{*}(2, R)$ subgroup of the boson-boson block of $G l\left(N_{f}+2 \mid 2\right)$ is a symmetry of the partition function. This symmetry is broken by the formation of a chiral condensate. With maximum breaking of chiral symmetry consistent with the Vafa-Witten theorem only an $\operatorname{SPp}\left(2 \mid N_{f}+2\right)$ subgroup remains unbroken. The Goldstone manifold is then given by the maximum Riemannian submanifold [57] of the coset space $G l\left(N_{f}+2 \mid 2\right) / O S p\left(2 \mid N_{f}+2\right)$ with fermionfermion integration domain given by $U\left(N_{f}+2\right) / O\left(N_{f}+2\right)$ and boson-boson integration domain equal to $U^{*}(2, R) / S p(2)$.

An explicit representation of this manifold is given by

$$
U=V^{T} G_{A} V
$$

where

$$
G_{A}=\left(\begin{array}{ccc}
1_{N_{f}+2} & 0 & 0 \\
0 & 0 & 1 \\
0 & -1 & 0
\end{array}\right)
$$

and an explicit parameterization of $V$ is given by

$$
V=\left(\begin{array}{ccc}
U_{F F} & \alpha_{1} & \alpha_{2} \\
\beta_{1} & a & b \\
\beta_{2} & c & d
\end{array}\right),
$$

where $U_{F F}$ is an $\left(N_{f}+2\right) \times\left(N_{f}+2\right)$ unitary matrix, $\alpha_{i}$ and $\beta_{i}$ are Grassmann variables and $a, b, c$ and $d$ are real numbers.

\section{The Scalar Susceptibility in ChPT}

In this section we compute the scalar susceptibility according to standard ChPT with $N_{f}$ sea quarks and no valence quarks. The scalar susceptibility is defined by

$$
K^{a b}=-\int d^{4} x d^{4} y \operatorname{Tr} t^{a}\left\langle x\left|\frac{1}{\not D+m}\right| y\right\rangle t^{b}\left\langle y\left|\frac{1}{\not D+m}\right| x\right\rangle
$$

For equal quark masses, the Dirac operator is flavor diagonal. Replacing the spatial integrals by a sum over the Dirac spectrum, we obtain

$$
K^{a b}=-\frac{\delta_{a b}}{2} \sum_{k} \frac{1}{\left(i \lambda_{k}+m\right)^{2}} .
$$


By introducing the isovector scalar source term $s^{a} \bar{q} t^{a} q$ the scalar susceptibility can be expressed in terms of a derivative of the QCD partition function (notice that the disconnected contribution vanishes in this case)

$$
K^{a b}=\partial_{s_{a}} \partial_{s_{b}} \log Z
$$

Alternatively, the low-energy limit of the scalar susceptibility can be obtained from the effective chiral partition function. To lowest order in the momenta and the quark masses this partition function is determined uniquely by the pattern of chiral symmetry breaking 61, 62,

$$
\int_{U \in G / H} d U e^{-\int d^{4} x\left[\frac{F_{\beta}^{2}}{4} \operatorname{Tr}\left(\partial_{\mu} U \partial_{\mu} U^{-1}\right)-\frac{\Sigma_{0}}{2} \operatorname{Tr}\left(\hat{\mathcal{M}}_{\beta}\left(U+U^{-1}\right)\right)\right]}
$$

The mass matrix also includes an isovector scalar source term. In all three cases, the matrix $U$ is parametrized as

$$
U=e^{i 2 \pi_{a} T^{a} / F_{\beta}}
$$

where the sum is over the generators $T^{a}$ of the coset (notice that in this representation the mass matrix is diagonal for all three cases). The pion decay constant $F_{\beta}$ is chosen such that we have the standard Gell-Mann-Oakes-Renner relation for all three cases with the convention $\operatorname{Tr} T^{a} T^{b}=\delta^{a b} / 2$. For the three cases under consideration the appropriate parameters are given in Table 1.

Table 1: The mass matrix and the pion decay constant for the three different patterns of chiral symmetry breaking.

\begin{tabular}{|c||c|c|c|}
\hline$G / H$ & $\hat{\mathcal{M}}_{\beta}$ & $F_{\beta}$ & $\beta$ \\
\hline$S U\left(N_{f}\right)$ & $m \mathbf{1}_{N_{f}}+s_{a} t^{a}$ & $F$ & 2 \\
$S U\left(2 N_{f}\right) / S p\left(2 N_{f}\right)$ & $\left(\begin{array}{c}\left(m \mathbf{1}_{N_{f}}+s_{a} t^{a}\right) / 2 \\
0 \\
S U\left(N_{f}\right) / O\left(N_{f}\right)\end{array}\right.$ & $F / \sqrt{2}$ & 1 \\
$\left(m \mathbf{1}_{N_{f}}+s_{a} t^{a}\right) / 2$ & & \\
& & $F / \sqrt{2}$ & 4 \\
\hline
\end{tabular}

To second order in the $\pi$ fields we find

$$
\frac{1}{2}\left(U+U^{-1}\right)=1-\frac{2}{F_{\beta}^{2}} \pi_{k} \pi_{l} T^{k} T^{l} .
$$

By differentiating the partition function with respect to the scalar source terms and calculating the loop integral we obtain

$$
\begin{aligned}
K^{a b} & =\frac{4 \Sigma^{2}}{F^{4}} \operatorname{Tr}\left(t^{a} T^{k} T^{l}\right) \operatorname{Tr}\left(t^{b} T^{m} T^{n}\right)\left\langle\int d^{4} x d^{4} y \pi_{k}(x) \pi_{l}(x) \pi_{m}(y) \pi_{n}(y)\right\rangle_{1-\text { loop }} \\
& =\frac{\Sigma^{2}}{16 \pi^{2} F^{4}} \operatorname{Tr}\left(t^{a}\left\{T^{k}, T^{l}\right\}\right) \operatorname{Tr}\left(t^{b}\left\{T^{k}, T^{l}\right\}\right) \log (\Lambda / m) .
\end{aligned}
$$


The only nontrivial task is to calculate the sum

$$
Q^{(\beta)}=\frac{1}{4} \operatorname{Tr}\left(t^{a}\left\{T^{k}, T^{l}\right\}\right) \operatorname{Tr}\left(t^{b}\left\{T^{k}, T^{l}\right\}\right)
$$

Let us first consider the case $\beta=2$. We already have seen that $K^{a b}$ is diagonal in isospin space. For the generators $t^{a}$ and $t^{b}$ we conveniently choose

$$
t^{a}=t^{b}=\frac{1}{2}\left(\delta_{i 1} \delta_{j 1}-\delta_{i 2} \delta_{j 2}\right) .
$$

The remaining diagonal generators are chosen as (consistent with the relation $\operatorname{Tr} t^{k} t^{l}=$ $\left.\frac{1}{2} \delta^{k l}\right)$,

$$
\operatorname{diag}\left(t_{D}^{n}\right)=(\underbrace{1, \cdots, 1}_{n},-n, \underbrace{0, \cdots, 0}_{N_{f}-n-1}) / \sqrt{2\left(n^{2}+n\right)},
$$

whereas the off-diagonal generators are given by

$$
t_{A}^{k l}=i\left(\delta_{i k} \delta_{j l}-\delta_{j k} \delta_{i l}\right) / 2
$$

and

$$
t_{S}^{k l}=\left(\delta_{i k} \delta_{j l}+\delta_{j k} \delta_{i l}\right) / 2
$$

Notice that for $\beta=2$ we do not distinguish between $T^{a}$ and $t^{a}$. For the sum $Q^{(2)}$ we thus obtain

$$
Q^{(2)}=\frac{1}{4} \sum_{n=2}^{N_{f}-1} \frac{1}{n(n+1)}+\frac{1}{16}\left(N_{f}-2\right)=\frac{1}{16} \frac{N_{f}^{2}-4}{N_{f}},
$$

where the first term in this equation is due to the diagonal generators and the second term of this equation is due to the off-diagonal generators.

As usual the case of $\beta=2$ is the simplest case. The next simplest case is $\beta=4$ with coset space given by $S U\left(N_{f}\right) / O\left(N_{f}\right)$. In the sum (44) the same diagonal generators enter as in the case of $\beta=2$. However, only the symmetric off-diagonal generators contribute to this sum in this case, so that the contribution from the off-diagonal generators is exactly half of what it was for $\beta=2$. This results in

$$
Q^{(4)}=\frac{1}{4} \sum_{n=2}^{N_{f}-1} \frac{1}{n(n+1)}+\frac{1}{32}\left(N_{f}-2\right)=\frac{1}{32} \frac{\left(N_{f}+4\right)\left(N_{f}-2\right)}{N_{f}} .
$$

Finally we consider the case $\beta=1$. In this case the generators of the coset have the structure 54

$$
T^{k}=\left(\begin{array}{cc}
A & B \\
B^{\dagger} & A^{T}
\end{array}\right)
$$


where $A$ is a Hermitian traceless matrix and $B^{T}=-B$ (both are $N_{f} \times N_{f}$ matrices). The sum over the generators is again calculated by distinguishing the diagonal generators from the off-diagonal generators. The diagonal generators are given by

$$
T_{D}^{a}=\frac{1}{\sqrt{2}}\left(\begin{array}{cc}
t_{D}^{a} & 0 \\
0 & t_{D}^{a}
\end{array}\right)
$$

where the $t_{D}^{a}$ are defined in (41). In order to satisfy the symmetry requirements, the off-diagonal generators have four nonzero entries in this case. Adding up all contributions we find

$$
Q^{(1)}=18 \frac{N_{f}-2}{N_{f}}+\frac{1}{8}\left(N_{f}-2\right)=\frac{1}{8} \frac{\left(N_{f}-2\right)\left(N_{f}+1\right)}{N_{f}}
$$

where the first term is the contribution from the diagonal generators and the second term is the contribution from the off-diagonal generators.

Our final result for $K^{a b}$ can be summarized as

$$
K^{a b}=-\frac{\Sigma^{2}}{32 \pi^{2} F^{4}} \frac{\left(N_{f}-2\right)\left(N_{f}+\beta\right)}{\beta N_{f}} \log (m / \Lambda) .
$$

With the same assumption for the $\lambda$ and $m$ dependence of the spectral density as in [4] we find that the slope of the Dirac spectrum at the origin is given by

$$
\rho^{\prime}(0)=\frac{\Sigma^{2}}{16 \pi^{2} F^{4}} \frac{\left(N_{f}-2\right)\left(N_{f}+\beta\right)}{\beta N_{f}} .
$$

The slope vanishes for $N_{f}=2$ for all three values of $\beta$. This is in agreement with instanton simulations 41] performed for the cases of $\beta=1$ and $\beta=2$.

\section{Partially Quenched Chiral Perturbation Theory}

The physics of QCD at low energies is completely determined by the spontaneous symmetry breaking pattern described above [61, 62, 63, 64]. In general, the effective Lagrangian of partially quenched QCD is based on the spontaneous chiral symmetry breaking pattern of the graded Lie-groups $G \rightarrow H$. The Goldstone fields are given by

$$
U=\exp \left(i 2 \Phi / F_{\beta}\right)
$$

where $F_{\beta}$ is the pion decay constant and $\Phi=\phi_{a} T^{a}$ with $T^{a}$ the generators of the maximum Riemannian submanifold of $G / H$ (notice that in this representation the mass matrix is diagonal for all three cases). We use the normalization $\operatorname{Str} T^{a} T^{b}=\frac{1}{2} \delta^{a b}$. To lowest order 
in the momenta and the quark masses, the Euclidean effective Lagrangian is obtained in the very same way as in Chiral Perturbation Theory [61, 62, 13, 9]. This results in

$$
\mathcal{L}_{\text {eff }}=\frac{F_{\beta}^{2}}{4} \operatorname{Str}\left(\partial_{\mu} U \partial_{\mu} U^{-1}\right)-\frac{\Sigma_{0}}{2} \operatorname{Str}\left(\hat{\mathcal{M}}_{\beta}\left(U+U^{-1}\right)\right)+m_{0}^{2} \Phi_{0}^{2}+\alpha \partial_{\mu} \Phi_{0} \partial_{\mu} \Phi_{0}
$$

The last two terms in (52) represent the mass and kinetic terms of the super- $\eta^{\prime}$ flavorsinglet field $\Phi_{0}=\operatorname{Str}(\Phi)$. They are introduced for the same symmetry reasons as in the three-color case [13, 9]. Below we are only interested in the case that $m_{0} \sim \Lambda_{Q C D}$. In that case the term proportional to $\alpha$ is sub-leading, and it will be ignored from now on. The effective partition function is given by

$$
Z_{\text {eff }}=\int_{U \in G / H} d U e^{-\int d^{4} x \mathcal{L}_{\text {eff }}}
$$

where the coset, the mass matrix and the pion decay constant for the three values of $\beta$ are given in the table below.

Table 2: The mass matrix and the pion decay constant for the three different patterns of chiral symmetry breaking for the partially quenched effective partition function.

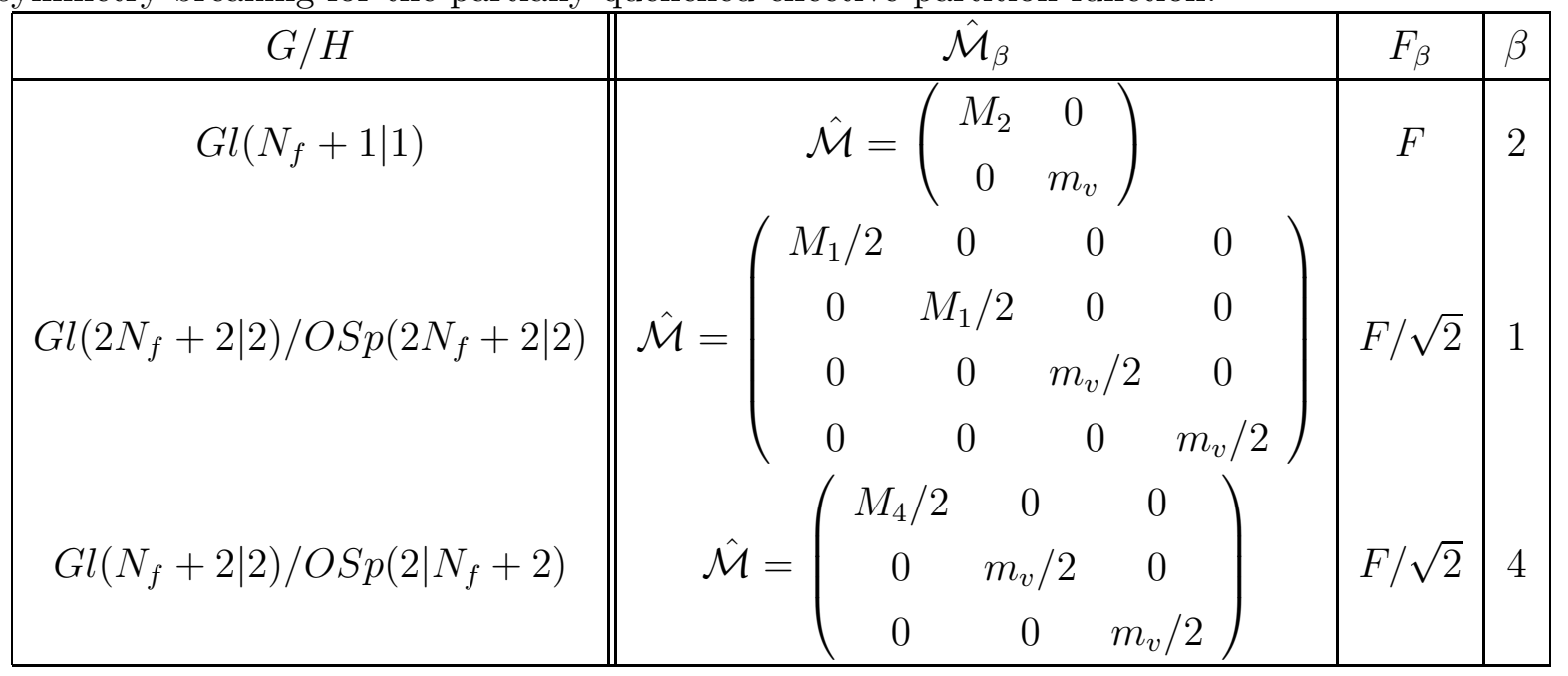

The mass matrix in the fermion-fermion block of the mass matrix $\mathcal{M}_{\beta}$ is given by

$$
\begin{aligned}
& M_{2}=\operatorname{diag}(\underbrace{m_{s}, \ldots, m_{s}}_{N_{f}}, m_{v}+J), \\
& M_{1}=\operatorname{diag}(\underbrace{m_{s}, \ldots, m_{s}}_{N_{f}}, m_{v}+J), \\
& M_{4}=\operatorname{diag}(\underbrace{m_{s}, \ldots, m_{s}}_{N_{f}}, m_{v}+J, m_{v}+J) .
\end{aligned}
$$

For the zero momentum component of the flavor singlet mass term, the same physical interpretation as in the three-color case holds [5]:

$$
\left\langle\nu^{2}\right\rangle=\frac{F_{\beta}^{2} m_{0}^{2} V}{2} .
$$


The factors in front of the first two terms in the effective Lagrangian (52) have been chosen such that the Goldstone fields are normalized in the usual way. The masses of the Goldstone bosons are given by the usual Gell-Mann-Oakes-Renner relation

$$
M_{i j}^{2}=\left(m_{i}+m_{j}\right) \Sigma_{0} / F^{2} .
$$

with quark condensate in the chiral limit denoted by $\Sigma_{0}$.

In the case of chiral perturbation theory for QCD it is possible to distinguish a domain where the partition function factorizes in a piece dominated by the zero momentum modes and a factor involving the nonzero momentum modes [63, 64]. The same is true for the partially quenched chiral Lagrangian. For valence quark masses below the Thouless energy given by [0, 25, 5]

$$
m_{c} \sim \frac{F^{2}}{\Sigma_{0} L^{2}},
$$

the fluctuations of the zero modes in the the effective partition function dominate the fluctuations of the nonzero momentum modes. Then the integral over the zero momentum modes factorizes from the partition function and the calculation of $\Sigma\left(m_{v}\right)$ is reduced to a group integral. This integral has been calculated explicitly for $\beta=2$ with the result that $\Sigma\left(m_{v}\right)$ is in complete agreement with chiral Random Matrix Theory.

\section{Calculation of $\Sigma\left(m_{v}\right)$ for $\beta=1$}

From a computational point of view, two-color pqChPT is not very different from three-color pqChPT [9]. To perform a calculation to one-loop order one has to construct the meson propagator. The effective Lagrangian discussed in previous section is based on the symmetric super-space $G l\left(2 N_{f}+2 \mid 2\right) / O S p\left(2 N_{f}+2 \mid 2\right)$. An explicit representation of $\Phi$ in (51) in terms of a $\left(2 N_{f}+4\right) \times\left(2 N_{f}+4\right)$ matrix field is given by

$$
\Phi=\left(\begin{array}{cccc}
\phi & \psi & \eta_{v} / 2 & \eta_{w} / 2 \\
\psi^{\dagger} & \phi^{T} & \chi_{v} / 2 & \chi_{w} / 2 \\
\chi_{v} / 2 & -\eta_{v} / 2 & i \tilde{\phi}_{v} / \sqrt{2} & i \tilde{\phi}_{v w} / 2 \\
\chi_{w} / 2 & -\eta_{w} / 2 & i \tilde{\phi}_{v w} / 2 & i \tilde{\phi}_{w} / \sqrt{2}
\end{array}\right),
$$

where the $\left(N_{f}+1\right) \times\left(N_{f}+1\right)$ ordinary matrices $\phi=\phi^{\dagger}$ and $\psi=-\psi^{T}$ contain the ordinary mesons made of quark and anti-quarks, the $N_{f}+1$-vectors $\chi_{v}, \chi_{w}, \eta_{v}$ and $\eta_{w}$ represent the fermionic mesons consisting of a ghost quark and an ordinary anti-quark, and finally the real fields $\tilde{\phi}_{v}, \tilde{\phi}_{w}$ and $\tilde{\phi}_{v w}$ are the mesons with two ghost quarks. With the proper 
normalization of the fields the Hermitian matrix field $\phi$ is given by

$$
\phi=\left(\begin{array}{ccc}
\phi_{1,1} / 2 & \cdots & \phi_{1, N_{f}+1} / 2 \sqrt{2} \\
\vdots & \ddots & \vdots \\
\phi_{1, N_{f}+1}^{*} / 2 \sqrt{2} & \cdots & \phi_{N_{f}+1, N_{f}+1} / 2
\end{array}\right)
$$

and the anti-symmetric matrix $\psi$ is given by

$$
\psi=\left(\begin{array}{cccc}
0 & \psi_{1,2} / 2 \sqrt{2} & \ldots & \psi_{1, N_{f}+1} / 2 \sqrt{2} \\
-\psi_{1,2} / 2 \sqrt{2} & \ddots & & \\
\vdots & & & \\
& & & \psi_{N_{f}, N_{f}+1} / 2 \sqrt{2} \\
-\psi_{1, N_{f}+1} / 2 \sqrt{2} & \ldots & -\psi_{N_{f}, N_{f}+1} / 2 \sqrt{2} & 0
\end{array}\right) .
$$

To second order in the pion fields there is no mixing between the off-diagonal Goldstone modes. However, because of the term $\operatorname{Str}^{2} \Phi$, the situation is more complicated for the diagonal Goldstone modes. The propagator of these modes is obtained by inverting the quadratic form in the effective Lagrangian obtained by expanding the $U$ fields up to second order. The mixing matrix of the diagonal mesons is given by

$$
M^{(1)}=\left(\begin{array}{ccccc}
2 & \cdots & 2 & -i \sqrt{2} & -i \sqrt{2} \\
\vdots & & \vdots & \vdots & \vdots \\
2 & \cdots & 2 & -i \sqrt{2} & -i \sqrt{2} \\
-i \sqrt{2} & \cdots & -i \sqrt{2} & -1 & -1 \\
-i \sqrt{2} & \cdots & -i \sqrt{2} & -1 & -1
\end{array}\right)
$$

The propagator of the diagonal mesons is thus given by

$$
G^{(1)}\left(p^{2}\right)=\left(\Gamma+m_{0}^{2} M^{(1)}\right)^{-1},
$$

where $\Gamma$ is diagonal with

$$
\Gamma_{i i}=\left\{\begin{array}{lcc}
p^{2}+M_{s s}^{2} & \text { for } & 1 \leq i \leq N_{f} \\
p^{2}+M_{v v}^{2} & \text { for } & N_{f}+1 \leq i \leq N_{f}+3,
\end{array}\right.
$$

The matrix $\Gamma+M^{(1)}$ can be inverted by expansion in a power series in $\Gamma^{-1} M^{(1)}$ and using the relation that

$$
\left(\Gamma^{-1} M^{(1)}\right)^{2}=2 N_{f}\left(p^{2}+M_{s s}^{2}\right)^{-1} \Gamma^{-1} M^{(1)}
$$

By re-summing the geometric series we obtain

$$
G^{(1)}\left(p^{2}\right)=\frac{1}{\Gamma}-\frac{1}{\left(p^{2}+M_{s s}^{2}\right)^{-1}+2 N_{f} m_{0}^{2}} m_{0}^{2} \frac{1}{\Gamma} M^{(1)} \frac{1}{\Gamma} .
$$


Below, we only need this propagator at the origin for $1 \leq i \leq N_{f}+1$ which is given by

$$
G_{i i}^{(1)}=\frac{1}{V} \sum_{p}\left[\frac{1}{p^{2}+M_{i i}^{2}}-\frac{2 m_{0}^{2}\left(p^{2}+M_{s s}^{2}\right)}{\left(p^{2}+M_{i i}^{2}\right)^{2}\left(p^{2}+M_{s s}^{2}+2 N_{f} m_{0}^{2}\right)}\right] .
$$

The quenched limit is obtained in the limit of infinite sea quark masses at fixed values of $m_{0}$ and $m_{v}$. Standard ChPT power counting rules are recovered when the super- $\eta^{\prime}$ decouples, that is when the singlet mass $m_{0}$ is sent to infinity. It is easy to check that in this case the propagator (68) in the sea quark sector is just the standard propagator in a quark basis with the singlet channel projected out. Finally, if both the valence and sea quark masses are set equal, we recover the standard result for two-color ChPT.

The valence quark mass dependence of the chiral condensate is computed as in the three color case [10, 5]. Since we are mainly interested in the non-analytic valence quark mass dependence of the chiral condensate, we will perform a one-loop computation, and we will ignore the analytic corrections coming from the $O\left(p^{4}\right)$ effective Lagrangian.

The usual tadpole diagrams have to be evaluated. At finite volume, the pion propagator at the origin is

$$
\Delta\left(M^{2}\right)=\frac{1}{V} \sum_{p} \frac{1}{p^{2}+M^{2}},
$$

where the sum is over the momenta in the box. In the thermodynamic limit the sum can be replaced by an integral resulting in (in four Euclidean dimensions)

$$
\Delta\left(M^{2}\right)=\frac{1}{16 \pi^{2}} M^{2} \ln \frac{M^{2}}{\Lambda^{2}}
$$

where $\Lambda$ is the cutoff.

It is now straightforward to calculate the valence quark mass dependence of the chiral condensate. Collecting the different contributions we find

$$
\begin{aligned}
\Sigma^{(1)}\left(m_{v}\right)= & \Sigma_{0}\left[1-\frac{1}{F^{2}}\left\{2 N_{f} \Delta\left(M_{v s}^{2}\right)+G_{v v}^{(1)}-2 \Delta\left(M_{v v}^{2}\right)\right\}\right] \\
= & \Sigma_{0}\left[1-\frac{1}{N_{f} F^{2}}\left\{2 N_{f}^{2} \Delta\left(M_{v s}^{2}\right)-\left(N_{f}+1\right) \Delta\left(M_{v v}^{2}\right)\right.\right. \\
& \left.\left.+\left(M_{s s}^{2}-M_{v v}^{2}\right) \partial_{M_{v v}^{2}} \Delta\left(M_{v v}^{2}\right)\right\}\right] .
\end{aligned}
$$

The first term in the first equation is due to the off-diagonal mesons consisting out of a valence quark and a sea quark, the second term is due to the diagonal meson consisting out of two valence quarks, and the last term is due to mesons consisting out of a bosonic and a fermionic quarks. As usual there is an additional minus sign for fermionic meson loops. 
7. Calculation of $\Sigma\left(m_{v}\right)$ for $\beta=4$

For fermions in the adjoint representation the Goldstone manifold is determined by the pattern of chiral symmetry breaking according to

$$
G l\left(N_{f}+2 \mid 2\right) \rightarrow O S p\left(2 \mid N_{f}+2\right) .
$$

The Goldstone manifold is given by the maximum Riemannian submanifold of $G l\left(N_{f}+\right.$ $2 \mid 2) / O S p\left(2 \mid N_{f}+2\right)$. The effective Lagrangian has exactly the same structure as for $\beta=1$, but in this case an explicit representation of the Goldstone modes is given by

$$
\Phi=\left(\begin{array}{ccccc}
A_{1,1} / \sqrt{2} & \cdots & A_{1, N_{f}+2} / 2 & \alpha_{1} / 2 & \beta_{1} / 2 \\
\vdots & \ddots & \vdots & \vdots & \vdots \\
A_{1, N_{f}+2} / 2 & \cdots & A_{N_{f}+2, N_{f}+2} / \sqrt{2} & \alpha_{N_{f}+2} / 2 & \beta_{N_{f}+2} / 2 \\
\beta_{1} / 2 & \cdots & \beta_{N_{f}+2} / 2 & i \phi_{1} / 2 & 0 \\
-\alpha_{1} / 2 & \cdots & -\alpha_{N_{f}+2} / 2 & 0 & i \phi_{1} / 2
\end{array}\right) .
$$

Here, $A$ is a real symmetric matrix, $\phi_{1}$ is a real variable and the $\alpha_{k}$ and $\beta_{k}$ are Grassmann variables.

In order to do a one-loop calculation we need an explicit expression for the pion propagator. Because of the singlet mass term the inversion of the quadratic form involving the diagonal mesons is nontrivial. The propagator of the diagonal mesons is given by

$$
G^{(4)}\left(p^{2}\right)=\left(\Gamma+m_{0}^{2} M^{(4)}\right)^{-1},
$$

where the mixing matrix is given by

$$
M^{(4)}=\left(\begin{array}{cccc}
1 & \cdots & 1 & -i \sqrt{2} \\
\vdots & & \vdots & \vdots \\
1 & \cdots & 1 & -i \sqrt{2} \\
-i \sqrt{2} & \cdots & -i \sqrt{2} & -2
\end{array}\right)
$$

and $\Gamma$ is diagonal with

$$
\Gamma_{i i}=\left\{\begin{array}{llc}
p^{2}+M_{s s}^{2} & \text { for } & 1 \leq i \leq N_{f}, \\
p^{2}+M_{v v}^{2} & \text { for } & N_{f}+1 \leq i \leq N_{f}+3 .
\end{array}\right.
$$

The inverse in (74) can be calculated simply by expanding the expression as a geometric series in $\Gamma^{-1} M^{(4)}$. If we use the relation

$$
\left(\Gamma^{-1} M^{(4)}\right)^{2}=N_{f} \frac{1}{p^{2}+M_{s s}^{2}} \Gamma^{-1} M^{(4)}
$$


the geometric series can be resummed as

$$
G^{(4)}\left(p^{2}\right)=\frac{1}{\Gamma}-\frac{1}{1+\left(p^{2}+M_{s s}^{2}\right)^{-1} N_{f} m_{0}^{2}} m_{0}^{2} \frac{1}{\Gamma} M^{(4)} \frac{1}{\Gamma} .
$$

Below we only need the first $N_{f}+2$ diagonal elements of the propagator matrix at the origin. They are given by

$$
G_{i i}^{(4)}=\frac{1}{V} \sum_{p}\left[\frac{1}{p^{2}+M_{i i}^{2}}-\frac{m_{0}^{2}\left(p^{2}+M_{s s}^{2}\right)}{\left(p^{2}+M_{i i}^{2}\right)^{2}\left(p^{2}+M_{s s}^{2}+N_{f} m_{0}^{2}\right)}\right] .
$$

The calculation of the valence quark mass dependence of the chiral condensate proceeds in exactly the same way as for $\beta=1$. As final answer we find

$$
\Sigma^{(4)}\left(m_{v}\right)=\Sigma_{0}\left[1-\frac{1}{2 F^{2}}\left\{N_{f} \Delta\left(M_{s v}^{2}\right)+2 G_{v v}^{(4)}-\Delta\left(M_{v v}^{2}\right)\right\}\right] .
$$

\section{Valence Quark Mass Dependence of the Chiral Condensate and the Dyson Index}

Before studying the valence quark mass dependence of the chiral condensate in five different limiting cases we write $\Sigma\left(m_{v}\right)$ as a single formula valid for all three different values of $\beta$.

The result for the valence quark mass dependence of the chiral condensate for gauge fields in the fundamental representation and three or more colors was obtained previously [10, 5]. In our present notation the result can be rewritten as

$$
\Sigma^{(2)}\left(m_{v}\right)=\Sigma_{0}\left[1-\frac{1}{F^{2}}\left\{N_{f} \Delta\left(M_{s v}^{2}\right)+G_{v v}^{(2)}-\Delta\left(M_{v v}^{2}\right)\right\}\right],
$$

where $G_{v v}^{(2)}=G_{v v}^{(4)}$. With Dyson index $\beta$ of the Dirac operator equal to $\beta=1$ for QCD with two colors and fermions in the fundamental representation, $\beta=2$ for three or more colors and fermions in the fundamental representation, and $\beta=4$ for two or more colors in the adjoint representation, the result for the three different cases considered above can be written as

$$
\Sigma^{(\beta)}\left(m_{v}\right)=\Sigma_{0}\left[1-\frac{1}{F^{2}}\left\{\frac{2 N_{f}}{\beta} \Delta\left(M_{s v}^{2}\right)+G_{v v}^{(\beta)}-\frac{2}{\beta} \Delta\left(M_{v v}^{2}\right)\right\}\right] .
$$

The diagonal propagator at the origin is given by

$$
G_{v v}^{(\beta)}=\frac{1}{V} \sum_{p}\left[\frac{1}{p^{2}+M_{v v}^{2}}-\frac{\left(1+\delta_{\beta, 1}\right) m_{0}^{2}\left(p^{2}+M_{s s}^{2}\right)}{\left(p^{2}+M_{i i}^{2}\right)\left(p^{2}+M_{s s}^{2}+N_{f}\left(1+\delta_{\beta, 1}\right) m_{0}^{2}\right)}\right] .
$$


Notice that this propagator becomes independent of $\beta$ in the limit $m_{0} \rightarrow \infty$ when the usual ChPT counting rules prevail. Previously, a similar unified description was obtained for sum-rules for the inverse eigenvalues of the Dirac operator [16, 56]. Below we will discuss five limiting cases for $\Sigma\left(m_{v}\right)$ obtained in (82).

i) In the limit $m_{v}=m_{s} \ll m_{0}$ we should recover the mass dependence of the chiral condensate in standard chiral perturbation theory. In this case with $M_{v v}=M_{s s}=M$, the diagonal propagator reduces to

$$
G_{v v}^{(\beta)}=\frac{N_{f}-1}{N_{f}} \Delta\left(M^{2}\right)
$$

and the expression for $\Sigma\left(m_{v}\right)$ can be written as

$$
\Sigma^{(\beta)}\left(m_{v}\right)=\Sigma_{0}\left[1-\frac{1}{F^{2}} \frac{\left(N_{f}-1\right)\left(1+2 N_{f} / \beta\right)}{N_{f}} \Delta\left(M^{2}\right)\right] .
$$

We observe that for $N_{f}=1$ the result is independent of the Dyson index $\beta$. Indeed, in this case the only axial symmetry is broken explicitly by the anomaly for all three values of $\beta$. The factor $\left(N_{f}-1\right)\left(1+2 N_{f} / \beta\right)$ exactly counts the number of Goldstone bosons which is equal to the number of generators of $S U\left(2 N_{f}\right) / S p\left(2 N_{f}\right)$ for $\beta=1$, the number of generators of $S U\left(N_{f}\right)$ for $\beta=2$, and the number of generators of $S U\left(N_{f}\right) / O\left(N_{f}\right)$ for $\beta=4$.

ii) Next we consider the case that both $m_{v} \ll m_{0}$ and $m_{s} \ll m_{0}$. In this case the diagonal propagator is given by

$$
G_{v v}^{(\beta)}=\frac{N_{f}-1}{N_{f}} \Delta\left(M_{v v}^{2}\right)-\frac{1}{N_{f}} M_{v v}^{2} \partial_{M_{v v}^{2}} \Delta\left(M_{v v}^{2}\right) .
$$

This results in the valence quark mass dependence

$$
\Sigma\left(m_{v}\right)=\Sigma_{0}\left[1-\frac{1}{F^{2}}\left\{\frac{2 N_{f}}{\beta} \Delta\left(M_{v s}^{2}\right)+\left(\frac{N_{f}-1}{N_{f}}-\frac{2}{\beta}\right) \Delta\left(M_{v v}^{2}\right)+\frac{1}{N_{f}}\left(M_{s s}^{2}-M_{v v}^{2}\right) \partial_{M_{v v}^{2}} \Delta\left(M_{v v}^{2}\right)\right\}\right] .
$$

In the thermodynamic limit, the singular part of $\Sigma\left(M_{v}\right)$ is then given by

$$
\Sigma\left(m_{v}\right)=\Sigma_{0}\left[1-\frac{\Sigma_{0}}{8 \pi^{2} N_{f} F^{4}}\left\{\frac{N_{f}^{2}}{\beta}\left(m_{v}+m_{s}\right) \log \frac{m_{v}+m_{s}}{2 \mu}+\left(m_{s}+\left(N_{f}\left(1-\frac{2}{\beta}\right)-2\right) m_{v}\right) \log \frac{m_{v}}{\mu}\right\}\right],
$$

where $\mu=\Lambda^{2} F^{2} / 2 \Sigma_{0}$. In the limit of $m_{s} \rightarrow 0$ this result simplifies to

$$
\Sigma\left(m_{v}\right)=\Sigma_{0}\left[1-\frac{\Sigma_{0}}{8 \pi^{2} N_{f} \beta F^{4}}\left(N_{f}-2\right)\left(N_{f}+\beta\right) m_{v} \log \frac{m_{v}}{\mu}\right] .
$$


iii) In the range $\lambda_{\text {min }} \ll m_{v} \ll 1 / L^{2} \Lambda_{\mathrm{QCD}} \ll m_{0}$, and $m_{s} \ll m_{v}$ the propagator at the origin (68) is dominated by the zero-momentum mode [63, 65, 5, 6] resulting in

$$
\Delta\left(M_{v v}^{2}\right) \sim \frac{1}{M_{v v}^{2} V}
$$

The valence quark mass dependence of the chiral condensate in the limit $m_{s} \ll m_{v}$ then reduces to

$$
\Sigma_{v} \sim \Sigma_{0}\left[1-\left(\frac{2 N_{f}}{\beta}+\frac{1}{2}-\frac{1}{\beta}\right) \frac{1}{m_{v} V \Sigma_{0}}\right] .
$$

iv) Next we consider the quenched limit which is obtained for $m_{s} \gg m_{v}$ and $m_{s} \gg m_{0}$. In this case the diagonal propagator at the origin is given by

$$
G_{v v}^{(\beta)}=\frac{1}{V} \sum_{p}\left[\frac{1}{p^{2}+M_{v v}^{2}}-\frac{\left(1+\delta_{\beta, 1}\right) m_{0}^{2}}{\left(p^{2}+M_{v v}^{2}\right)^{2}}\right] .
$$

This results in the valence quark mass dependence

$$
\begin{aligned}
\Sigma\left(m_{v}\right) & =\Sigma_{0}\left[1-\frac{1}{F^{2}}\left\{(1-2 / \beta) \Delta\left(M_{v v}^{2}\right)+\left(1+\delta_{\beta, 1}\right) m_{0}^{2} \partial_{M_{v v}^{2}} \Delta\left(M_{v v}^{2}\right)\right\}\right] \\
& \sim \Sigma_{0}\left[1-\frac{1}{16 \pi^{2} F^{2}}\left(1+\delta_{\beta, 1}\right) m_{0}^{2} \log \frac{m_{v}}{\mu}\right]+O\left(m_{v}\right) .
\end{aligned}
$$

where we have only quoted the infrared singular terms. We find that the chiral condensate diverges for $m_{v} \rightarrow 0$.

In the domain $m_{v} \ll m_{s}$ the expression (88) is identical to the quenched result (93) provided $\left(1+\delta_{\beta, 1}\right) m_{0}^{2}=M_{s s}^{2} / N_{f}$. This is in accordance with the Ward identity relating the sea quark mass and the topological susceptibility $\left\langle\nu^{2}\right\rangle=F_{\beta}^{2} M_{s s}^{2} V / 2 N_{f}\left(1+\delta_{\beta, 1}\right)$ 66, and the relation (57).

v) In lattice QCD with staggered fermions the Lagrangian does not possess the axial $U(1)$ symmetry at finite lattice spacing (although it should be recovered in the continuum limit). A detailed discussion of quenched lattice simulations for the valence quark mass dependence of the chiral condensate in this case was given in [67]. The effective partition function is then given by (53) with an effective Lagrangian without singlet terms. The diagonal propagator at the origin is thus given by

$$
G_{v v}=\frac{1}{V} \sum_{p} \frac{1}{p^{2}+M_{v v}^{2}}
$$

in the cases i), ii) and iv). In the quenched case, the term $\sim m_{0}^{2}$ is absent and the $O\left(m_{v}\right)$ terms have to be taken into account. We then find

$$
\Sigma\left(m_{v}\right)=\Sigma_{0}\left[1-\frac{(1-2 / \beta) \Sigma_{0}}{8 \pi^{2} F^{4}} m_{v} \log \frac{m_{v}}{\mu}\right] .
$$

For $\beta=2$ the coefficient of the chiral logarithm vanishes and higher order corrections become important 68 . 


\section{Spectral Density in the Ergodic and Diffusive Domains}

In the ergodic regime, for valence quark masses $m_{v} \ll F^{2} / \Sigma_{0} L^{2}$ the microscopic spectral density is given by chRMT [7], whereas for $\Lambda_{\mathrm{QCD}} \gg m_{v} \gg \lambda_{\min } \sim 1 / L^{4} \Sigma_{0}$ the one-loop calculation of $\Sigma\left(m_{v}\right)$ is valid. In this section we calculate the spectral densities in each of these domains. We first show that in the ergodic domain the one-loop result for $\Sigma\left(m_{v}\right)$ coincides with the chRMT result.

The QCD partition function for two colors and fundamental fermions belongs to the chGOE $(\beta=1)$ universality class [16]. In a sector of topological charge $|\nu|$ and in the chiral limit, the result reads [69, 70]:

$$
\begin{aligned}
\rho_{s}^{\operatorname{chGOE}}(u)= & \frac{u}{2}\left[J_{2 N_{f}+|\nu|}^{2}(u)-J_{2 N_{f}+|\nu|+1}(u) J_{2 N_{f}+|\nu|-1}(u)\right] \\
& +\frac{1}{2} J_{2 N_{f}+|\nu|}(u)\left[1-\int_{0}^{u} d t J_{2 N_{f}+|\nu|}(t)\right] .
\end{aligned}
$$

The QCD Dirac operator with adjoint fermions belongs to the chGSE $(\beta=4)$ universality class. In this case the microscopic spectral density is given by [71, 80

$$
\begin{aligned}
\rho_{s}^{\mathrm{chGSE}}(u)= & u\left[J_{N_{f}+2|\nu|}^{2}(2 u)-J_{N_{f}+2|\nu|+1}(2 u) J_{N_{f}+2|\nu|-1}(2 u)\right] \\
& -\frac{1}{2} J_{2 N_{f}+2|\nu|}(2 u) \int_{0}^{2 u} d t J_{N_{f}+2|\nu|}(t) .
\end{aligned}
$$

For completeness we also give the microscopic spectral density for the chGUE ( $\beta=$ 2) [72, 41] which describes the Dirac operator of QCD with three or more colors and fundamental fermions,

$$
\rho_{s}^{\mathrm{chGUE}}(u)=\frac{u}{2}\left[J_{N_{f}+|\nu|}^{2}(u)-J_{N_{f}+|\nu|+1}(u) J_{N_{f}+|\nu|-1}(u)\right] .
$$

The valence quark mass dependence of the chiral condensate in terms of the microscopic variable $x=m_{v} V \Sigma_{0}$ is obtained via the relation

$$
\frac{\Sigma(x)}{\Sigma_{0}}=\int_{0}^{\infty} d u \frac{2 x}{u^{2}+x^{2}} \rho_{s}(u)+\frac{|\nu|}{x},
$$

where the last term is the contribution from the exactly zero eigenvalues of the Dirac operator [22]. In the case of the chGUE this integral can be perform analytically resulting in

$$
\begin{aligned}
\frac{\Sigma(x)}{\Sigma_{0}} & =x\left[I_{N_{f}+|\nu|}(x) K_{N_{f}+|\nu|}(x)+I_{N_{f}+|\nu|+1}(x) K_{N_{f}+|\nu|-1}(x)\right]+\frac{|\nu|}{x} \\
& =1-\frac{N_{f}+|\nu|}{x}+\frac{|\nu|}{x}+\cdots=1-\frac{N_{f}}{x}+\cdots .
\end{aligned}
$$


The asymptotic result for $x \gg 1$ for the chGOE and the chGSE can be easily derived using this result and the asymptotic result of the remaining terms. The leading $1 / x$ correction can be obtained by expanding before integration. The only additional integral that is required is

$$
\int_{0}^{\infty} J_{\mu}(x)=1
$$

The asymptotic result in terms of the Dyson index is given by

$$
\frac{\Sigma_{v}(x)}{\Sigma_{0}}=1-\frac{4 N_{f}+\beta-2}{2 \beta x},
$$

which coincides with the result (91) obtained from chiral perturbation theory. Notice, that the leading order asymptotic correction is independent of $\nu$. This is no longer true for higher order corrections and a summation over all $\nu$ is required to compare with results obtained for fixed value of $\theta$ (which is zero in our case) [73.

In the diffusive domain, for $1 / L^{2} \Lambda_{\mathrm{QCD}} \ll m_{v} \ll \lambda_{\mathrm{QCD}}$ the valence quark mass dependence of the chiral condensate is given by the one-loop result (88). The spectral density is given by the discontinuity of $\Sigma\left(m_{v}\right)$ across the imaginary axis $(\rho(\lambda) / V=$ Disc $\left.\left.\right|_{m_{v}=i \lambda} \Sigma\left(m_{v}\right) / 2 \pi\right)$ [5, 6]. As final result we then find for the spectral density of the Dirac operator with $N_{f}$ flavors with quark masses $m_{s} \ll \lambda \ll \Lambda_{\mathrm{QCD}}$

$$
\begin{aligned}
& \frac{\rho(\lambda)}{V}=\frac{\Sigma_{0}}{\pi}\left[1+\frac{\Sigma_{0}}{16 \pi^{2} N_{f} F^{4}}\left\{\frac{2 N_{f}^{2}}{\beta}|\lambda| \operatorname{Arctg} \frac{|\lambda|}{m_{s}}-\left(N_{f}\left(\frac{2}{\beta}-1\right)+2\right) \pi|\lambda|\right.\right. \\
& \left.\left.-\frac{N_{f}^{2}}{\beta} m_{s} \log \frac{\lambda^{2}+m_{s}^{2}}{\mu^{2}}-2 m_{s} \log \frac{|\lambda|}{\mu}\right\}\right] .
\end{aligned}
$$

The spectral density has a logarithmic infrared divergence when $m_{s} \neq 0$. In the limit $m_{s} \ll \lambda$, the expression (104) reduces to:

$$
\frac{\rho(\lambda)}{V}=\frac{\Sigma_{0}}{\pi}\left[1+\frac{\left(N_{f}-2\right)\left(N_{f}+\beta\right) \Sigma_{0}}{16 \pi \beta N_{f} F^{4}}|\lambda|\right] .
$$

The result for the slope for $\beta=2$ was first obtained in [4]. Remarkably, the slope vanishes for $N_{f}=2$ for three values of $\beta$. This is in agreement with instanton liquid simulations of the QCD Dirac spectrum 41.

In the quenched case, the spectral density is found to be:

$$
\frac{\rho^{Q}(\lambda)}{V}=\frac{\Sigma_{0}}{\pi}\left[1-\frac{\left(1+\delta_{\beta, 1}\right) m_{0}^{2}}{16 \pi^{2} F^{2}} \log \frac{|\lambda|}{\mu}\right] .
$$

We find a logarithmic divergence of the spectral density for all three values of $\beta$. This result is consistent with quenched instanton liquid and lattice QCD simulations [42, 74]. on liquid simulations and lattice QCD simulations [42, 74. For staggered fermions away 
from the continuum limit this term has to be modified according to the discussion given in $\mathrm{v}$ ) of the previous section. For the spectral density we then find

$$
\frac{\rho^{Q}(\lambda)}{V}=\frac{\Sigma_{0}}{\pi}\left[1-\frac{\Sigma_{0}}{16 \pi F^{4}}\left(1-\frac{2}{\beta}\right)|\lambda|\right] .
$$

\section{Conclusions}

We have shown that the infrared limit of the QCD Dirac spectrum is completely determined by the pattern of chiral symmetry breaking. The spectral density follows from the low energy effective partition function of the partially quenched QCD partition function which in addition to the usual quarks contains valence quarks and their bosonic superpartners. In this paper we have analyzed the case of two colors with fundamental fermions and the case of fermions in the adjoint representation. In both cases the effective Lagrangian is based on a Riemannian super-manifold originating from chiral symmetry breaking to an ortho-symplectic graded Lie Group. As is the case for QCD with three or more colors with fundamental fermions the Goldstone manifold is characterized by a symbiosis of compact and noncompact degrees of freedom.

Our one-loop calculation of the valence quark mass dependence of the chiral condensate for these two chiral symmetry breaking patterns completes an earlier calculation for QCD with three colors and fundamental fermions [5]. Its discontinuity results in an explicit expression for the spectral density of the Dirac operator valid for $\lambda \ll \Lambda_{\mathrm{QCD}}$. In all three cases we can distinguish two different domains in the Dirac spectrum which are separated by the Thouless energy. For valence quark masses below the Thouless energy the kinetic term decouples from the effective Lagrangian and the the one-loop result for the valence quark mass dependence of the chiral condensate is in complete agreement with chiral Random Matrix Theory. Agreement with chiral Random Matrix Theory to all orders has only been shown for $\beta=2$ by the means of an exact calculation of the superintegrals. It would be interesting to perform this calculation for the mathematically much more complicated cases of $\beta=1$ and $\beta=4$ as well. For valence quark masses beyond the Thouless energy the kinetic term in the partially quenched effective partition function has to be taken into account. The results for the spectral density in this domain are consistent with the results for the scalar susceptibility obtained with standard ChPT. In particular we have obtained an analytical expression for the slope of the Dirac spectrum. Remarkably, the slope vanishes for two massless flavors in all three cases. 


\section{Acknowledgements}

This work was partially supported by the US DOE grant DE-FG-88ER40388. One of us (D.T.) was supported in part by "Holderbank"-Stiftung. A. Altland, P. Damgaard, M.

Göckeler, J. Osborn, P. Rakow, A. Smilga, M. Stephanov, T. Wettig and M. Zirnbauer are acknowledged for useful discussions.

\section{References}

[1] T. Banks and A. Casher, Nucl. Phys. B169 (1980) 103.

[2] C. DeTar, Quark-gluon plasma in numerical simulations of QCD, in Quark gluon plasma 2, R. Hwa ed., World Scientific 1995.

[3] A.V. Smilga, Phys. Rep. 291, (1997) 1.

[4] A. Smilga and J. Stern, Phys. Lett. B318 (1993) 531.

[5] J.C. Osborn, D. Toublan and J.J.M. Verbaarschot, Nuc. Phys. B540 (1999) 317.

[6] P.H. Damgaard, J.C. Osborn, D. Toublan and J.J.M. Verbaarschot, hep-th/9811212, to be published in Nuc. Phys. B.

[7] J.J.M. Verbaarschot, Phys. Lett. B368 (1996) 137.

[8] A. Morel, J. Physique 48 (1987) 1111.

[9] C. Bernard and M. Golterman, Phys. Rev. D49 (1994) 486; C. Bernard and M. Golterman, hep-lat/9311070.

[10] M.F.L. Golterman and K.C. Leung, hep-lat/9711033; M.F.L. Golterman, Acta Phys. Polon. B25 (1994).

[11] S. Chandrasekharan, Nucl. Phys. Proc. Suppl. 42 (1995) 475; S. Chandrasekharan and N. Christ, Nucl. Phys. Proc. Suppl. 42 (1996) 527; N. Christ, Nucl. Phys. B (Proc. Suppl.) 53 (1997) 253.

[12] J.J.M. Verbaarschot, in Nonperturbative Approaches to Quantum Chromodynamics, D. Diakonov, ed., Gatchina 1995.

[13] C. Bernard and M. Golterman, Phys. Rev. D46 (1992) 853; M. Golterman, heplat/9411005, Chiral Perturbation Theory and the quenched approximation of $Q C D ; \mathrm{S}$. Sharpe, Phys. Rev. D46 (1992) 3146. 
[14] S. Sharpe, Phys. Rev. D 56 (1997) 7052.

[15] E.V. Shuryak and J.J.M. Verbaarschot, Nucl. Phys. A560 (1993) 306.

[16] J. Verbaarschot, Phys. Rev. Lett. 72 (1994) 2531; Phys. Lett. B329 (1994) 351.

[17] E. Brézin, S. Hikami and A. Zee, Nucl. Phys. B464 (1996) 411.

[18] G. Akemann, P. Damgaard, U. Magnea and S. Nishigaki, Nucl. Phys. B 487[FS] (1997) 721. (1994) 487.

[19] A.D. Jackson, M.K. Sener and J.J.M. Verbaarschot, Nucl. Phys. B479 (1996) 707.

[20] T. Guhr and T. Wettig, Nucl. Phys. B506 (1997) 589.

[21] A.D. Jackson, M.K. Sener and J.J.M. Verbaarschot, Nucl. Phys. B506 (1997) 612.

[22] P.H. Damgaard, Phys. Lett. B424 (1998) 322; B425 (1998) 151; G. Akemann and P.H. Damgaard, Phys. Lett. B432 (1998) 390; Nuc. Phys. B528 (1998) 411.

[23] P.H. Damgaard and S.M. Nishigaki, Phys. Rev. D57 (1998) 5299.

[24] J. Christiansen, hep-th/9809194.

[25] J.C. Osborn and J.J.M. Verbaarschot, Phys. Rev. Lett. 81 (1998) 268.

[26] M.A. Halasz and J.J.M. Verbaarschot, Phys. Rev. Lett. 74 (1995) 3920; M.A. Halasz, T. Kalkreuter and J.J.M. Verbaarschot, Nucl. Phys. Proc. Suppl. 53 (1997) 266.

[27] M.E. Berbenni-Bitsch, S. Meyer, A. Schäfer, J.J.M. Verbaarschot and T. Wettig, Phys. Rev. Lett. 80 (1998) 1146.

[28] M.E. Berbenni-Bitsch, M. Göckeler, T. Guhr, A.D. Jackson, J.Z. Ma, S. Meyer, A. Schäfer, H.A. Weidenmüller, T. Wettig and T. Wilke, Phys. Lett. B438 (1998) 14.

[29] J.Z. Ma, T. Guhr and T. Wettig, Eur. Phys. J. A2 (1998) 87.

[30] T. Guhr, J.Z. Ma, S. Meyer and T. Wilke, Phys. Rev. D59 (1999) 054501.

[31] M.E. Berbenni-Bitsch, S. Meyer and T. Wettig, Phys. Rev. D58 (1998) 071502.

[32] K. Splittorff and A.D. Jackson, hep-lat/9805018; K. Splittorf, hep-th/9810248.

[33] R. Pullirsch, K. Rabitsch, T. Wettig and H. Markum, Phys. Lett. B427 (1998) 119.

[34] P.H. Damgaard, U.M. Heller, A. Krasnitz and T. Madsen, Phys. Lett. B440 (1998) 129. 
[35] P.H. Damgaard, U.M. Heller and A. Krasnitz, hep-lat/981006.

[36] M. Göckeler, H. Hehl, P.E.L. Rakow, A. Schäfer and T. Wettig, Phys. Rev. D59 (1999) 094503.

[37] B.A. Berg, H. Markum, R. Pullirsch, hep-lat/9812010.

[38] F. Farchioni, I. Hip, C.B. Lang and M. Wohlgenannt, hep-lat/9812018.

[39] R.G. Edwards, U.M. Heller and R. Narayanan, hep-lat/9902021.

[40] R.G. Edwards, U.M. Heller, J. Kiskis and R. Narayanan, hep-th/9902117.

[41] J.J.M. Verbaarschot, Nucl. Phys. B427 (1994) 534.

[42] J.C. Osborn and J.J.M. Verbaarschot, Nucl. Phys. B525 (1998) 738.

[43] A. Altland and B.D. Simons, cond-mat/9811134.

[44] K. Takahashi and S. Iida, hep-th/9903119.

[45] B.L. Altshuler, I.Kh. Zharekeshev, S.A. Kotochigova and B.I. Shklovskii, Zh. Eksp. Teor. Fiz. 94 (1988) 343.

[46] H.A. Weidenmüller, Nucl. Phys. A 518 (1990) 1.

[47] K. Johnson, L. Lellouch and J. Polonyi, Nucl. Phys. B367 (1991) 675.

[48] R. Janik, G. Papp, M. Nowak and I.Zahed, Phys. Rev. Lett. 81 (1999) 264.

[49] K.B. Efetov, Adv. Phys. 32 (1983) 53; K.B. Efetov, Supersymmetry in disorder and chaos, Cambridge University Press, (1997).

[50] T. Guhr, A. Müller-Groeling and H.A. Weidenmüller, Phys. Rept. 299 (1998) 189.

[51] G. Montambaux, in Quantum Fluctuations, Les Houches, Session LXIII, E. Giacobino, S. Reynaud and J. Zinn-Justin, eds., Elsevier Science, 1995, condmat/9602071.

[52] F. Wegner, Z. Phys. B 49 (1983) 297.

[53] J. Verbaarschot, H. Weidenmüller, and M. Zirnbauer, Phys. Rep. 129 (1985) 367.

[54] M.E. Peskin, Nucl. Phys. B175 (1980) 197.

[55] S. Dimopoulos, Nucl. Phys. B168 (1980) 69; M. Vysotskii, Y. Kogan and M. Shifman, Sov. J. Nucl. Phys. 42 (1985) 318; D.I. Diakonov and V.Yu. Petrov, Lecture notes in physics, 417, Springer 1993. 
[56] A. Smilga and J.J.M. Verbaarschot, Phys. Rev. D51 (1995) 829.

[57] M. Zirnbauer, J. Math. Phys. 37 (1996) 4986.

[58] J.J.M. Verbaarschot, Lectures given at APCTP - RCNP Joint International School on Physics of Hadrons and QCD, Osaka, Japan, 1998 and the 1998 YITP Workshop on QCD and Hadron Physics, Kyoto, Japan, 1998, hep-ph/9902394.

[59] C. Vafa and E. Witten, Nucl. Phys. B234 (1984) 173.

[60] M.A. Halasz and J.J.M. Verbaarschot, Phys. Rev. D52 (1995) 2563.

[61] J. Gasser and H. Leutwyler, Ann. Phys. 158, 142 (1984); J. Gasser and H. Leutwyler, Nucl. Phys. B250, 465 (1985).

[62] H. Leutwyler, Ann. Phys. 235 (1994) 165.

[63] J. Gasser and H. Leutwyler, Phys. Lett. 188B(1987) 477; Nucl. Phys. B307 (1988) 763.

[64] H. Leutwyler and A. Smilga, Phys. Rev. D46 (1992) 5607.

[65] P. Hasenfratz and H. Leutwyler, Nucl. Phys. B343 (1990) 241.

[66] M. Shifman, A. Vainshtein and V. Zakharov, Nucl. Phys. B166 (1980) 493.

[67] M.E. Berbenni-Bitsch, M. Göckeler, H. Hehl, S. Meyer, P.E.L. Rakow, A. Schäfer and T. Wettig, hep-lat/9901013.

[68] P.E.L. Rakow, private communication.

[69] J.J.M. Verbaarschot, Nuc. Phys. B426 [FS] (1994) 559.

[70] T. Wettig, Habilitationsschrift Universität Heidelberg (1998).

[71] T. Nagao and P.J. Forrester, Nucl. Phys. B435 (1995) 401.

[72] J. Verbaarschot and I. Zahed, Phys. Rev. Lett. 70, 3852 (1993).

[73] P.H. Damgaard, hep-th/9903096.

[74] U. Sharan and M. Teper, hep-lat/9808017; hep-lat/9812009. 